\title{
Strongly coupled surface plasmons on thin shallow metallic gratings
}

\author{
Z. Chen, I. R. Hooper, and J. R. Sambles* \\ Thin Film Photonics Group, School of Physics, University of Exeter, Exeter EX4 4QL, United Kingdom
}

(Received 21 January 2008; published 17 April 2008)

\begin{abstract}
The optical response of a thin metallic film with shallow corrugations on both surfaces is explored and the structure is found to support a strongly coupled surface plasmon polariton when transverse magnetic radiation is incident in a plane parallel to the grating grooves. Modeling confirms that this strongly excited mode is the short range surface plasmon polariton and its presence is confirmed experimentally in the visible part of the spectrum.
\end{abstract}

DOI: 10.1103/PhysRevB.77.161405

PACS number(s): 73.20.Mf, 42.70.Qs, 78.20.Ci, 78.66.Bz

A surface plasmon polariton (SPP) is a fundamental electromagnetic excitation which may exist at the interface between a metal and a dielectric. ${ }^{1}$ On a flat metal surface, to optically excite the bound SPP mode, the in-plane momentum of the incident radiation must be suitably increased often by using a coupling prism as in the $\mathrm{Otto}^{2}$ or Kretschmann-Raether ${ }^{3}$ geometry or by employing a surface relief grating. ${ }^{4-6}$

For an optically opaque metal film, there is only one SPP mode, the single-interface surface plasmon polariton (SISPP). ${ }^{7}$ However, for thinner symmetrically bound metal films $(<100 \mathrm{~nm})$, the two SISPPs combine to form two coupled SPPs, the long range surface plasmon polariton (LRSPP) and the short range surface plasmon polariton (SRSPP). The LRSPP mode has an asymmetric charge distribution between the top and bottom surfaces with the electric field predominantly normal to the surface inside the metal. Conversely, the SRSPP has a charge distribution which is symmetric between the top and bottom surfaces with the electric field essentially parallel to the surface. Resonant coupling of incident photons to the LRSPP and the SRSPP modes on ${\text { both } \text { planar }^{8-12} \text { and corrugated thin metal films }}^{13,14}$ has been the subject of several studies. Excitement in this area was rekindled by the observation of substantially enhanced optical transmission through metallic films with a periodic array of subwavelength size holes by Ebbesen and co-workers. ${ }^{15-17}$ This enhancement may arise through the excitation of surface plasmons on either side of the metal film, ${ }^{15,16}$ suggesting that coupled SPP modes may be important.

Over the past 100 years, transverse magnetic (TM) excited SPPs in the "classical" mount (azimuth angle $\varphi=0^{\circ}$ ) have been extensively investigated. Apart from the study by Watts et al. ${ }^{18}$ of very deep metallic gratings, there have been almost no experimental or theoretical efforts undertaken to explore the SPP excited by TM polarized radiation in the "conical" mount (azimuth angle $\varphi=90^{\circ}$ ). In this study, strong coupling to a SPP mode is observed for TM polarized radiation incident on a shallow thin silver grating at $\varphi=90^{\circ}$.

Figure 1 shows the sample geometry; the azimuth angle $\varphi$ is fixed to $90^{\circ}$ with the grating grooves parallel to the plane of incidence. The experimental sample is prepared by first depositing a $23 \mathrm{~nm}$ silver film onto a silica grating with a pitch of $\lambda_{g}=252 \mathrm{~nm}$. This structure is then made optically symmetric by use of index matching fluid to couple two silica prisms symmetrically to the silver grating on its silica substrate. The surface profiles $y_{1}(x)$ and $y_{2}(x)$ of the top (in- cident) and bottom (transmitting) surfaces are given by

$$
y_{1}(x)=a \sin \left(k_{g} x\right)+d, \quad y_{2}(x)=a \sin \left(k_{g} x\right),
$$

where the two surfaces are separated by a mean thickness $d$, and $a$ is the amplitude of the grating, with $k_{g}=2 \pi / \lambda_{g}$ being the grating vector, parallel to the $x$ axis.

A grating may redirect incident photons into different channels: zero-order reflection $(R)$ and transmission $(T)$, diffracted order reflections and transmissions, and absorption $(A)$. In this study, for the wavelengths explored, the grating is nondiffractive. Then, to obtain the experimental absorption spectra, the wavelength-dependent reflection and transmission data are measured, and the absorption, which is a direct measure of the coupling strength, is acquired according to energy conservation $(A=1-R-T)$. Figure 2 illustrates the experimental TM polarized wavelength-dependent reflection (open squares), transmission (open triangles), and absorption (open stars) data together with the model results (dashed, dotted, and solid lines) for light incident on a thin silver grating with a pitch of $\lambda_{g}=252 \mathrm{~nm}$ at polar angles of $\theta$ $=65.0^{\circ}$ and $\varphi=90^{\circ}$. The theoretical method employed here has been reported extensively elsewhere ${ }^{19}$ and is based upon a combination of a scattering matrix approach and a coordinate transformation technique proposed by Chandezon et

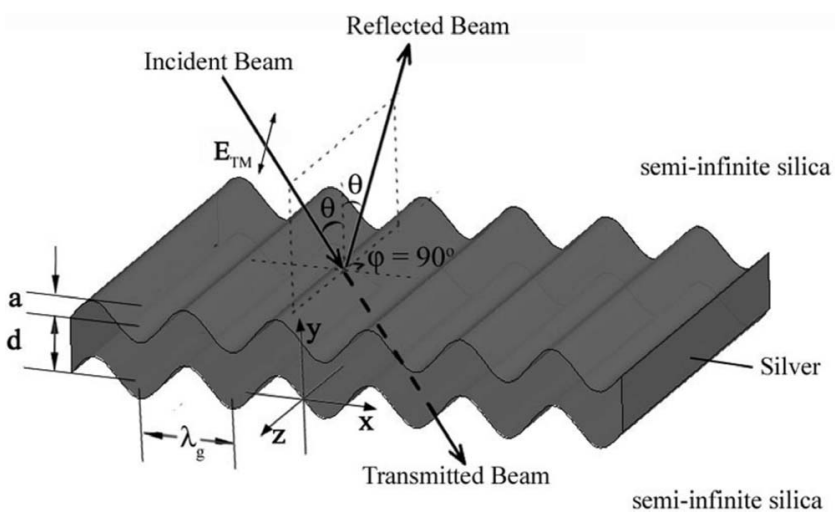

FIG. 1. Schematic diagram illustrating the sample and coordinate system used in this study. Here, $\lambda_{g}$ is the grating pitch, $a$ is the grating amplitude, $d$ is the thickness of the silver layer, $\theta$ is the polar angle, and $\varphi$ is the azimuth angle which is set to $90^{\circ}$. The silver layer is bounded by silica media. The orientation of the electric field $\left(\mathbf{E}_{\mathrm{TM}}\right)$ for TM polarization at $\varphi=90^{\circ}$ is also shown. 


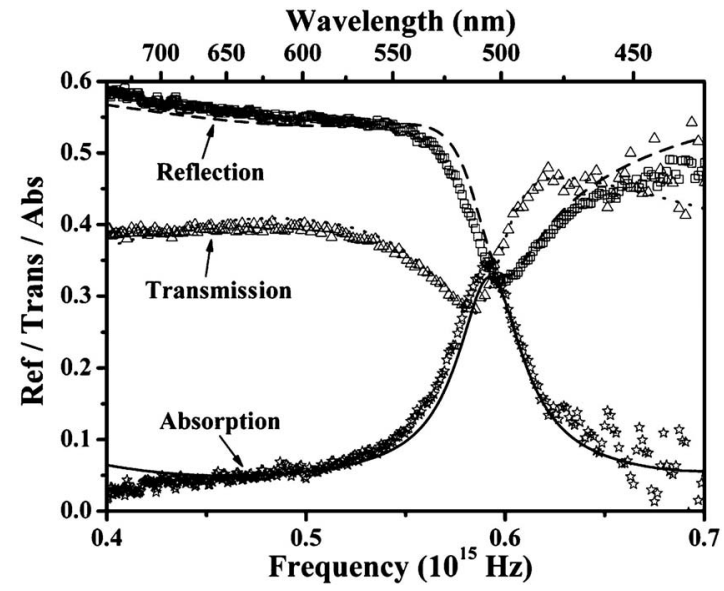

FIG. 2. Experimental reflection (open squares), transmission (open triangles), and absorption (open stars) together with the model fits (dashed, dotted, and solid lines) when TM polarized light is incident on a thin silver sinusoidal grating with a thickness of $23 \mathrm{~nm}$, a pitch of $252 \mathrm{~nm}$, and an amplitude of $8 \mathrm{~nm}$ at $\theta=65.0^{\circ}$ and $\varphi=90^{\circ}$.

$a l .,{ }^{20}$ which allows for easy matching of the electromagnetic boundary conditions at the interface. This computational approach has been shown to agree well with experimental data in the past. ${ }^{18,21,22}$ To model the silver across the visible spectrum, both the real and imaginary parts of its permittivity are described by polynomials fitted to experimentally derived values, ${ }^{23}$

$$
\begin{gathered}
\varepsilon_{r}=-255.3185+198.63 \omega-60.794 \omega^{2} \\
+8.381 \omega^{3}-0.43004 \omega^{4}, \\
\varepsilon_{i}=83.2575-132.79 \omega+90.474 \omega^{2}-32.88 \omega^{3}+6.6591 \omega^{4} \\
-0.70893 \omega^{5}+0.030913 \omega^{6},
\end{gathered}
$$

where $\omega=[2 \pi c / \lambda] \times 10^{-15} \mathrm{~s}^{-1}$. These polynomials inevitably pertain to somewhat different silver to that explored here in thin film form, but they are a good enough approximation to allow adequate modeling. Because of this and for simplicity in fitting data to a model, the refractive index of the bounding silica is treated as nondispersive with $n_{\text {silica }}=1.459$. In Fig. 2, the best comparison between the model and the experimental data is achieved when the thickness of the silver film and the amplitude are set to 23 and $8 \mathrm{~nm}$, respectively. Note that at a resonant frequency $f=0.595 \times 10^{15} \mathrm{~Hz}$, a significant absorption peak is readily observed in Fig. 2.

As mentioned in the single-interface deep grating work of Watts et al. ${ }^{18}$ the coupling of TM radiation (wavelength $\lambda_{0}$ ) to the SPP at $\varphi=90^{\circ}$ arises because of the difference in path length $\left(4 \pi a / \lambda_{0} \cos \theta\right)$ experienced by a photon striking the top of the groove compared to one impinging near the trough. Since the phase shift of the incident radiation is proportional to the amplitude, for a relatively shallow singleinterface grating coupling to the TM SPP mode at $\varphi=90^{\circ}$ should be very weak. However, in our experiments, as shown in Fig. 2, strong coupling is observed for a shallow thin film grating. To more clearly demonstrate that the coupling (a)

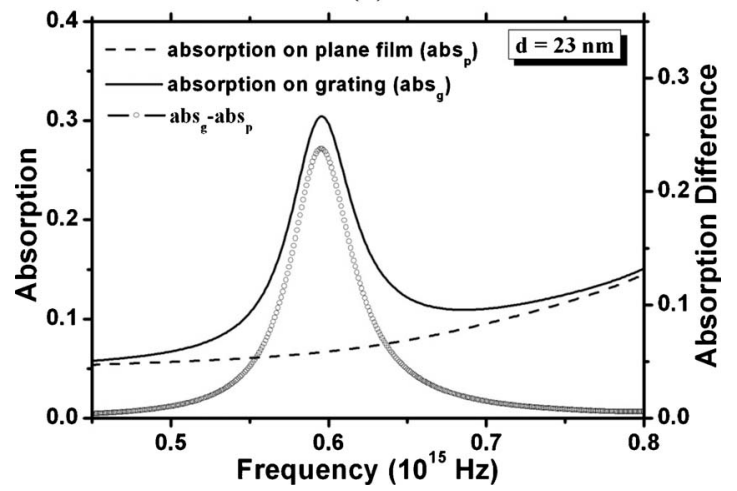

(b)

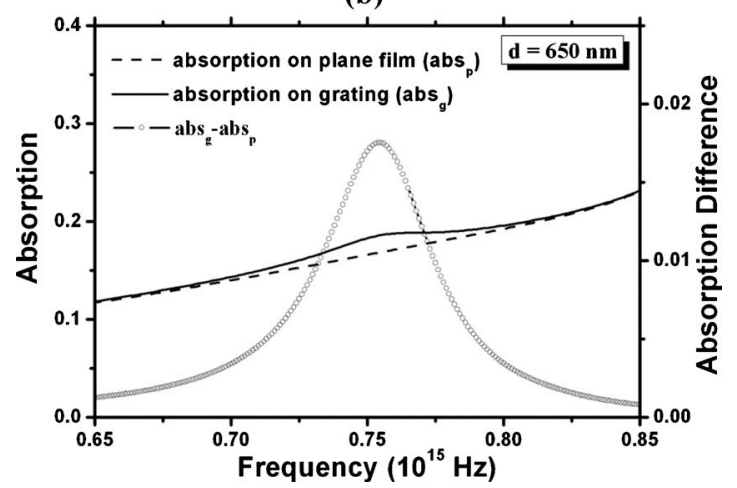

FIG. 3. Theoretical absorption at $\theta=65.0^{\circ}$ and $\varphi=90^{\circ}$ for (a) a $23 \mathrm{~nm}$ and (b) a $650 \mathrm{~nm}$ silver film with corrugation (solid line) and without corrugation (dashed line) together with the absorption difference (open circles). Lines without symbols are associated with the left axis and those with symbols with the right axis. (The grating has a pitch of $252 \mathrm{~nm}$ and an amplitude of $8 \mathrm{~nm}$.)

strength for the thin film grating case has been greatly increased, the theoretical TM polarized absorptions at polar angles $\theta=65.0^{\circ}$ and $\varphi=90^{\circ}$ for both plane and corrugated silver films with two different thicknesses $d=23$ and $650 \mathrm{~nm}$ are plotted in Fig. 3, where the corrugation in both cases has a pitch of $252 \mathrm{~nm}$ and an amplitude of $8 \mathrm{~nm}$. Also plotted in Fig. 3 is the absorption difference between the corrugated case and the noncorrugated case. Obviously, for a silver film with a thickness $d=650 \mathrm{~nm}$ and an amplitude $a=8 \mathrm{~nm}$, the incident radiation cannot "sense" the bottom surface and the structure should be identical to a single shallow silica and/or silver interface. As expected, Fig. 3(b) shows that for a single-interface shallow silver grating, the coupling strength (the height of the absorption difference peak) of the mode (the SISPP) at a resonant frequency $f=0.754 \times 10^{15} \mathrm{~Hz}$ is as small as $\sim 1.8 \%$. In contrast to the thin film case, the coupling strength at a resonant frequency $f=0.595 \times 10^{15} \mathrm{~Hz}$ is $\sim 23.8 \%$, as shown in Fig. 3(a). The coupling strength at the resonant frequency for the thin film shallow grating case has been enhanced by $0.238 / 0.018 \approx 13$ times compared to the single-interface shallow grating case.

Now, we shall identify the mode observed at the resonant frequency $f=0.595 \times 10^{15} \mathrm{~Hz}$. To do so, it is instructive to investigate the optical fields. Figure 4 shows the instantaneous $E_{x y}$ vector fields for such a strongly coupled SPP 


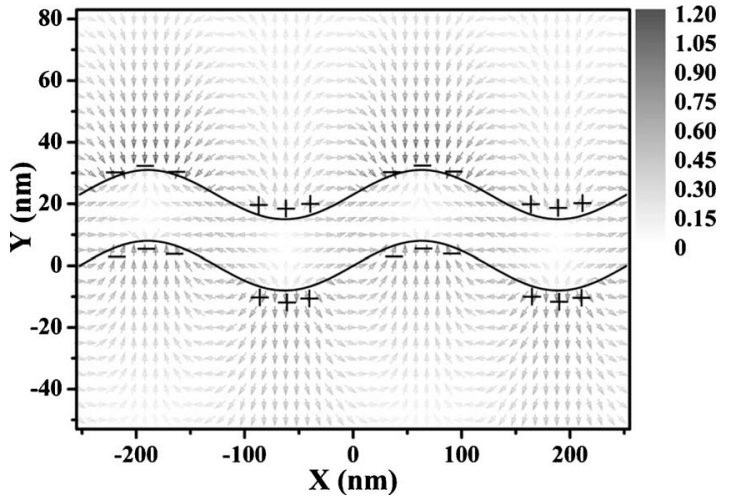

FIG. 4. Instantaneous $E_{x y}$ vector fields at a resonant frequency of $f=0.595 \times 10^{15} \mathrm{~Hz}$ for a thin silver film grating $\left(d=23 \mathrm{~nm}, \lambda_{g}\right.$ $=252 \mathrm{~nm}$, and $a=8 \mathrm{~nm}$ ) at $\theta=65.0^{\circ}$ and $\varphi=90^{\circ}$. The continuous lines are the surfaces of the thin metal grating. The grayscale of the arrows represents the magnitude of the $E_{x y}$ fields.

mode. Comparing the surface charge density on the top surface to that on the bottom surface, it clearly has a symmetric charge density distribution showing that the mode at $f$ $=0.595 \times 10^{15} \mathrm{~Hz}$ is the SRSPP mode. Also note that the surface charge density maxima on both the top and bottom surfaces locate at the grating peaks and troughs; at these positions, there are maximum normal components of electric field for TM polarization. In the conical mount and on excitation of the SPP, there will be two surface waves propagating along the metal surface with propagation angles of $\pm \Psi$. These two surface waves may be coupled together via two successive $k_{g}$ scattering events, setting up a standing wave in the $x$ direction and propagating along the $z$ direction. The nature of this standing wave in the $x$ direction is readily shown in Fig. 4, where the period of the standing wave is the same as the grating pitch.

It is known that as the film thickness decreases, the SISPP mode splits into two coupled SPP modes: the SRSPP and the LRSPP. Figure 5 shows the theoretical TM polarized resonant absorption $(A=1-R-T)$ as a function of frequency and thickness at $\theta=65.0^{\circ}$ and $\varphi=90^{\circ}$ for a corrugated silver film of $8 \mathrm{~nm}$ amplitude and $252 \mathrm{~nm}$ pitch. Note that since the

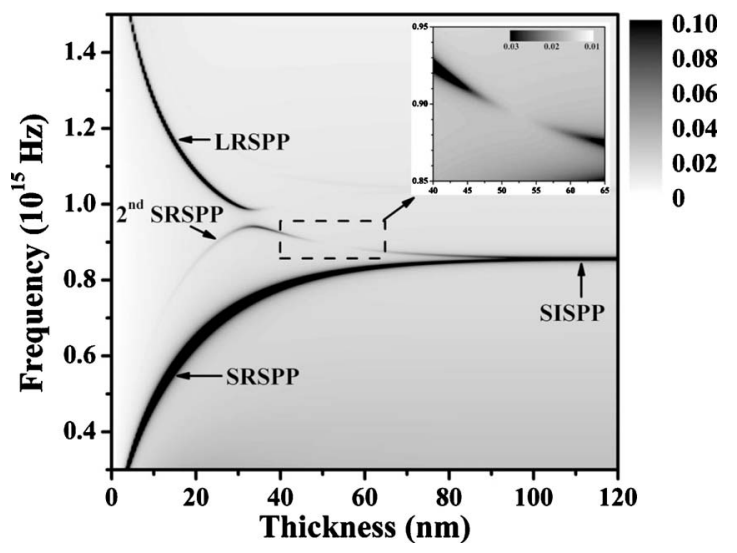

FIG. 5. Theoretical TM polarized absorption as a function of frequency and silver film thickness at $\theta=65.0^{\circ}$ and $\varphi=90^{\circ}$ for a corrugated silver film of $8 \mathrm{~nm}$ amplitude and $252 \mathrm{~nm}$ pitch. (a)

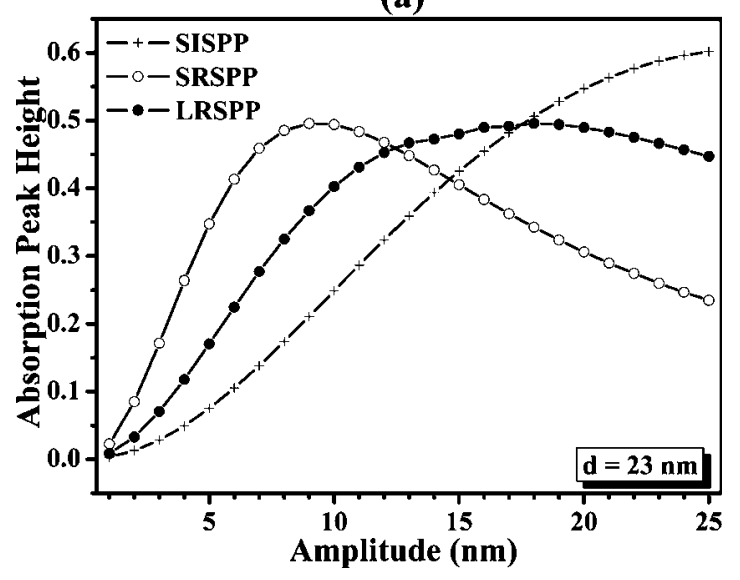

(b)

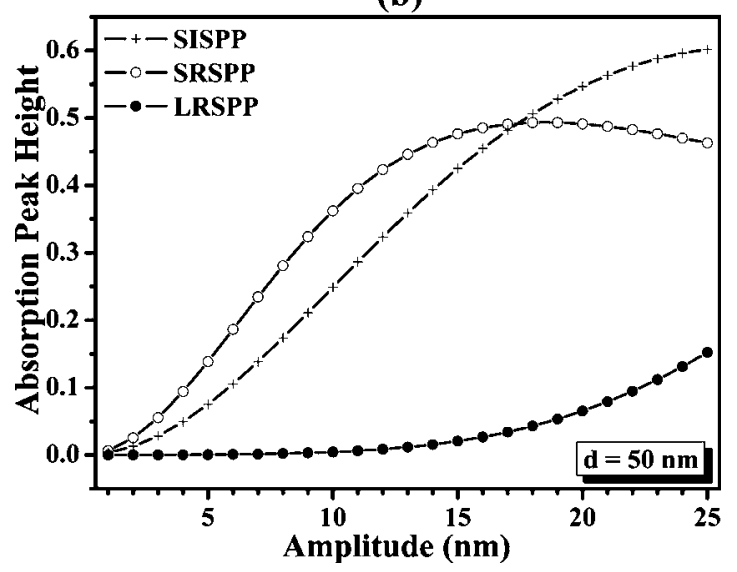

(c)

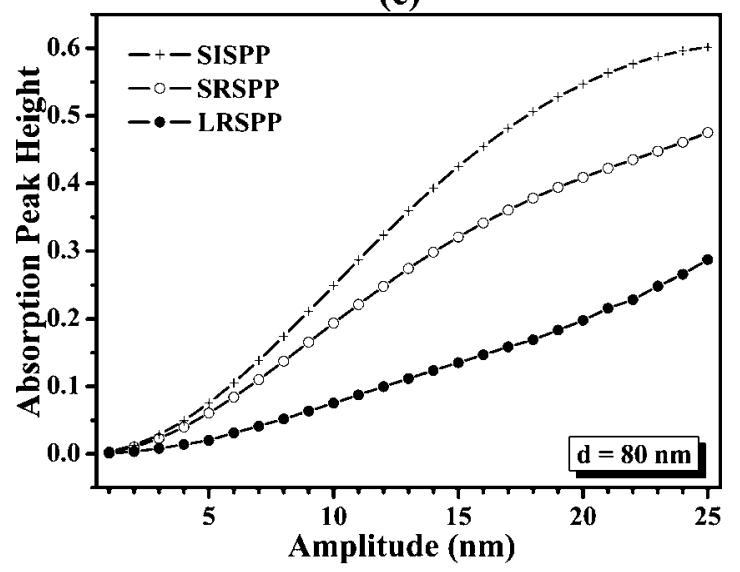

FIG. 6. Theoretical TM excited SRSPP (open circles) and LRSPP (solid circles) absorption peak height as a function of amplitude at $\theta=65.0^{\circ}$ and $\varphi=90^{\circ}$ for different film thicknesses (a) $d$ $=23 \mathrm{~nm}$, (b) $d=50 \mathrm{~nm}$, and (c) $d=80 \mathrm{~nm}$. The absorption peak height for the TM excited SISPP (crosses) is also plotted in each of the graphs.

LRSPP has now moved out of the visible region, the dielectric constant of silver which was described as polynomials above is now replaced by a Drude model with a plasma frequency $\omega_{p}=1.32 \times 10^{16} \mathrm{~s}^{-1}$ and a relaxation time $\tau=1.45$ $\times 10^{-14} \mathrm{~s}$. As can be seen from Fig. 5, when the thickness of the silver film $d>100 \mathrm{~nm}$, only the SISPP mode giving an 
absorption peak is excited. As the thickness decreases, the SISPP splits into the SRSPP and the LRSPP, with the frequency separation of these two coupled modes dominated by the thickness of the silver film. Again, one sees clearly from Fig. 5 that the coupling strength of the SRSPP has been enhanced compared to that of the SISPP, with the coupling strength of the SRSPP itself increasing with decreasing film thickness. Also note that the second order SRSPP shifts down rapidly in resonant frequency with decreasing film thickness, and in the small thickness region, it is observed to interfere with the LRSPP to form a significant anticrossing centered around $d \approx 33 \mathrm{~nm}$ and $f \approx 0.95 \times 10^{15} \mathrm{~Hz}$.

Interestingly, Fig. 5 also shows that with decreasing thickness, the coupling strength for the LRSPP first decreases to a minimum (almost zero) at $d \approx 52 \mathrm{~nm}$, and then increases again, as shown more clearly in the inset. This minimum arises because of interference between the incident fields and the reflected fields (from both the incident surface and the transmitted surface) making at some thicknesses the resulting fields look like the fields in the plane thin film case.

To more clearly demonstrate the TM excited SRSPP resonant absorption enhancement on a thin silver grating for $\varphi$ $=90^{\circ}$, it is instructive to investigate the coupling strength for the SRSPP and the LRSPP at different film thicknesses and amplitudes. Figure 6 shows the TM excited SRSPP (open circles) and the TM excited LRSPP (solid circles) absorption peak heights (coupling strength) as a function of the amplitude for different film thicknesses $d=80,50$, and $23 \mathrm{~nm}$ at $\theta=65.0^{\circ}$ and $\varphi=90^{\circ}$. For comparison, the TM excited SISPP (crosses) absorption peak height is also plotted. As shown in Fig. 6(c), for a thin silver grating with a thickness of $80 \mathrm{~nm}$, the absorption for the SRSPP, the LRSPP, and the SISPP increases with increasing amplitude. At each amplitude, the absorption for both the SRSPP and the LRSPP is less than that for the SISPP. At $d=80 \mathrm{~nm}$, no absorption enhancement occurs for the SRSPP compared to the SISPP. When the thickness is $50 \mathrm{~nm}$, as shown in Fig. 6(b), in the amplitude region $0 \mathrm{~nm}<a<17 \mathrm{~nm}$, the absorption for the SRSPP is slightly higher than that for the SISPP. On further decrease in the film thickness to $23 \mathrm{~nm}$, a significant absorption enhancement for the SRSPP is readily observed in Fig. 6(a) in the amplitude region of $0 \mathrm{~nm}<a<14 \mathrm{~nm}$. Also note that for the amplitude region $0 \mathrm{~nm}<a<17 \mathrm{~nm}$ the absorption for the LRSPP is enhanced compared to the absorption for the SISPP. By comparing these plots in Fig. 6, it is found that the phenomenon of the SRSPP absorption enhancement observed in our experiments is a special case, which only occurs for a thin film grating with relatively shallow corrugations.

In conclusion, the first observation of strong coupling to a TM excited SPP is observed experimentally on a thin silver grating with shallow corrugations in the conical mount. By exploring the optical field distributions at the resonant frequency, this SPP mode is identified as the SRSPP mode. It is found that the coupling strength to the SRSPP mode is enhanced greatly compared to that of the TM excited SISPP mode on a single-interface grating with the same pitch and corrugation. Another interesting phenomenon, that the coupling strength of the TM excited LRSPP has an almost zero minimum with decreasing film thickness, is also reported.

Z.C. acknowledges funding from the Overseas Research Studentship Awards Scheme and the University of Exeter.

\footnotetext{
*j.r.sambles@exeter.ac.uk

${ }^{1}$ H. Raether, Surface Plasmons on Smooth and Rough Gratings (Springer-Verlag, Berlin, 1988).

${ }^{2}$ A. Otto, Z. Phys. 216, 398 (1968).

${ }^{3}$ E. Kretschmann and H. Raether, Z. Naturforsch. A 23, 2135 (1968).

${ }^{4}$ R. W. Wood, Philos. Mag. 4, 396 (1902).

${ }^{5}$ R. H. Ritchie, E. T. Arakawa, J. J. Cowan, and R. N. Hamm, Phys. Rev. Lett. 21, 1530 (1968).

${ }^{6}$ M. B. Sobnack, W. C. Tan, N. P. Wanstall, T. W. Preist, and J. R. Sambles, Phys. Rev. Lett. 80, 5667 (1998).

${ }^{7}$ J. Schoenwald, E. Burstein, and J. M. Elson, Solid State Commun. 12, 185 (1973).

${ }^{8}$ H. Boersch, J. Geiger, A. Imbusch, and N. Niedrig, Phys. Lett. 22, 146 (1966).

${ }^{9}$ A. Otto, Z. Phys. 219, 227 (1969).

${ }^{10}$ D. Sarid, Phys. Rev. Lett. 47, 1927 (1981).

${ }^{11}$ G. I. Stegeman, J. J. Burke, and D. G. Hall, Appl. Phys. Lett. 41, 906 (1982).

${ }^{12}$ R. K. Hickernell and D. Sarid, J. Opt. Soc. Am. B 3, 1059 (1986).

${ }^{13}$ T. Inagaki, M. Motosuga, E. T. Arakawa, and J. P. Goudonnet,
}

Phys. Rev. B 32, 6238 (1985).

${ }^{14}$ S. Dutta Gupta, G. V. Varada, and G. S. Agarwal, Phys. Rev. B 36, 6331 (1987).

${ }^{15}$ T. W. Ebbesen, H. J. Lezec, H. F. Ghaemi, T. Thio, and P. A. Wolff, Nature (London) 391, 667 (1998).

${ }^{16}$ H. F. Ghaemi, T. Thio, D. E. Grupp, T. W. Ebbesen, and H. J. Lezec, Phys. Rev. B 58, 6779 (1998).

${ }^{17}$ T. J. Kim, T. Thio, T. W. Ebbesen, D. E. Grupp, and H. J. Lezec, Opt. Lett. 24, 256 (1999).

${ }^{18}$ R. A. Watts, T. W. Preist, and J. R. Sambles, Phys. Rev. Lett. 79, 3978 (1997).

${ }^{19}$ N. P. K. Cotter, T. W. Preist, and J. R. Sambles, J. Opt. Soc. Am. A 12, 1097 (1995).

${ }^{20}$ J. Chandezon, M. T. Dupuis, G. Cornet, and D. Maystre, J. Opt. Soc. Am. 72, 839 (1982).

${ }^{21}$ Z. Chen, I. R. Hooper, and J. R. Sambles, New J. Phys. 9, 251 (2007).

${ }^{22}$ G. P. Bryan-Brown, M. C. Jory, S. J. Elston, and J. R. Sambles, J. Mod. Opt. 40, 959 (1993).

${ }^{23}$ Handbook of Optical Constants of Solids, edited by E. D. Palik (Academic, New York, 1985). 